\title{
Malignant spread of haemangioblastoma: report on two cases
}

\author{
J. MOHAN, BET TY B R O W ELL, A N D D. R. OPPENHEIMER \\ From the Departments of Neurosurgery and Neuropathology, \\ Radcliffe Infirmary, Oxford, and the Department of Neuropathy, \\ Frenchay Hospital, Bristol
}

SYNOPSIS Two cases are described in which, after successful removal of a cerebellar haemangioblastoma followed by several years of freedom from symptoms, there developed a progressive spinal cord compression, leading to death. At necropsy the spinal cords in both cases, and the brainstem in one case, were irregularly plastered with haemangioblastoma. Although there was no doubt that malignant spread had occurred from one or more primary tumours, the histology of the tumour tissue was in no way different from that of a conventional haemangioblastoma.

The cases which we are reporting here contradict the general belief that cerebellar haemangioblastomas are invariably benign.

\section{CASE 1}

D. M. Male. Age at death, 38 years.

FIRST ADMISSION September 1964, to Department of Neurosurgery, Radcliffe Infirmary, Oxford. The patient was admitted with six months' history of headache, apathy, unsteady gait, and tendency to fall to the right. Examination showed slurring dysarthria and coarse nystagmus on right gaze. The family history was not contributory. The blood haemoglobin was 12.8 $\mathrm{g} / \mathrm{dl}$. Carotid angiography showed slight ventricular enlargement. A pneumoencephalogram showed tonsillar herniation, displacement of the fourth ventricle to the left, and forward displacement of the aqueduct. Posterior fossa craniectomy revealed a cystic tumour containing golden fluid in the inferior part of the right cerebellar hemisphere. A cherry-red nodule $2 \frac{1}{2} \mathrm{~cm}$ in diameter was found in the cyst wall and removed completely. The patient made a full recovery and was able to go back to his job as a carpenter. He was followed up as an outpatient.

(Accepted 19 January 1976.)
SECOND ADMISSION October 1972. He had complained of headache, back pain, and left sciatica for 12 months. Examination showed papilloedema, unsteadiness on standing, limitation of back movement, and of straight leg raising to $50^{\circ}$. Vertebral angiography showed a small vascular mass in front of and on the right of the pons. A pneumoencephalogram showed obstruction in the prepontine region and enlarged ventricles. Myelography showed dilatation of the lumbosacral subarachnoid space. In view of the ventricular enlargement, a right ventriculoatrial shunt was performed. Postoperatively the sciatica disappeared. He was discharged and followed up as an outpatient.

THIRD ADMISSION May 1973. He complained of weakness of the left arm and of both legs. There was complete loss of joint position sense and stereognosis in the left arm and hand, but preservation of pain, temperature, and vibration sense. A brain scan was normal. On myelography, xanthochromic CSF was obtained, with partial holdup of contrast medium at T6 and complete hold-up at T4 vertebral levels. Spinal angiography revealed two vascular masses, one in front of the cord at $\mathrm{C} 2$ and one behind at C5-6 (Fig. 1). Cervical laminectomy from C2 to C7 was carried out on 29 May 1973. Three or four large extradural vessels were seen. When the dura mater was opened the posterior aspect of the cord was 


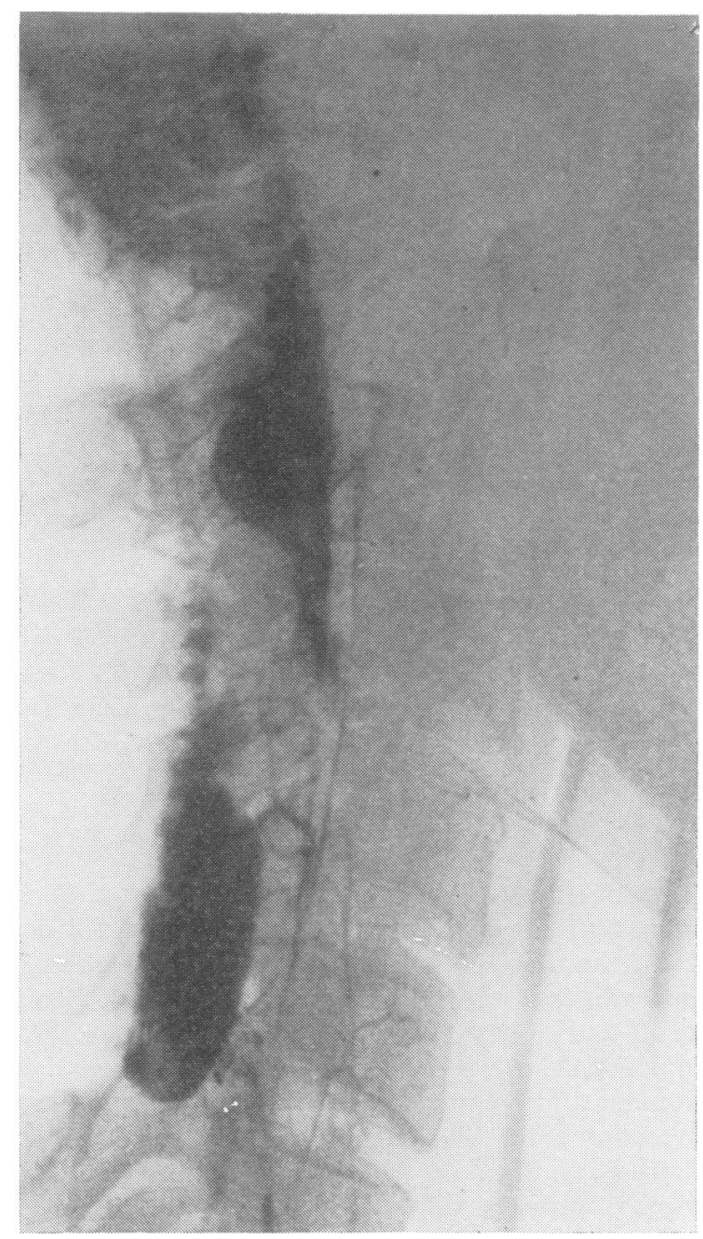

FIG. 1 Case 1 Spinal angiogram, showing two vascular masses, one anteriorly placed opposite the body of C2 vertebra, the other posteriorly placed opposite C5 vertebral body.

seen to be covered by very large vascular channels, in the middle of which was a red tumour which appeared to be merging with the cord. This was supplied by very large pulsating blood vessels. Above and below the tumour the subarachnoid space was present and filled with CSF, but in the region of the tumour the subarachnoid space was obliterated. Lateral slits were made in the dura mater, and the dura was left open. Postoperatively there was improvement in the joint position sense and stereognosis of the left arm.
FOURTH ADMISSION July 1973. The patient had been complaining of weakness in both arms. Examination confirmed weakness of arms and hands, diminished tendon reflexes, and absent joint position sense of fingers and hands. It was felt that the posteriorly situated cervical tumour was responsible for the greater part of his disability. On 9 July 1973 the cervical laminectomy was reopened and a posterior tumour at C5-6 vertebral level was removed. Postoperatively he was moving all four limbs and passing urine spontaneously. Just before his transfer to the Radiotherapy Department he was able to walk, although both upper limbs were still weak. Deep $x$ rays to a total dose of $3000 r$ were given to cervical and thoracic spine. He improved slowly until September 1974 when he developed bronchopneumonia. Soon after this he died.

NECROPSY FINDINGS There was a confluent bronchopneumonia, affecting both lower lobes. Otherwise, the viscera were unremarkable; in particular, there were no primary or metastatic tumours, vascular malformations, or cystic lesions.

The brain (1490 g) was not swollen, and the ventricles were of normal size. There was a Pudenz valve, with a catheter in the right lateral ventricle. The right cerebellar hemisphere showed surgical damage and was adherent to scar tissue deep to an occipital decompression. The cerebellar tonsils were soft and haemorrhagic, in the herniated position. The base of the brain was stained brown by iron pigment. The entire brainstem was

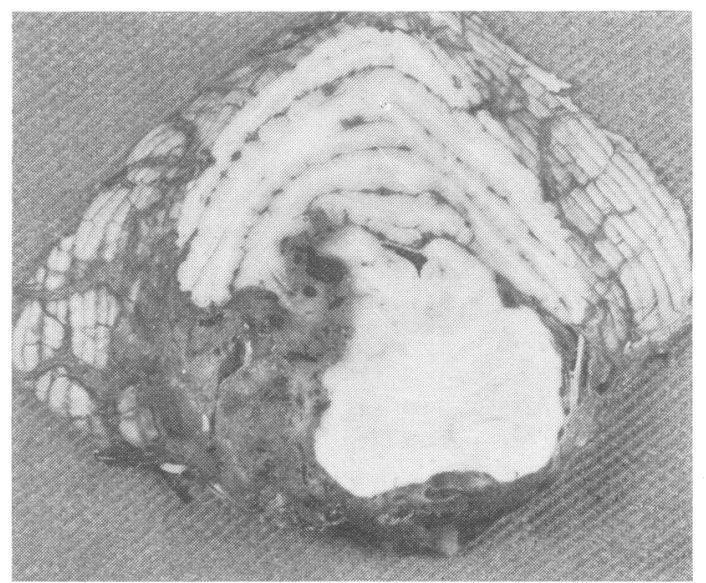

FIG. 2 Case 1. Upper pons, distorted and eroded by tumour, mainly on the right side (left of picture). 
invested in a layer of firm, dark tumour tissue, extending upwards into the lateral fissures, and surrounding the optic chiasm. The greatest bulk of tumour was in the right cerebellopontine angle, where is reached a thickness of $1 \frac{1}{2} \mathrm{~cm}$ (Fig. 2), distorting the brainstem and displacing it to the left, in places eroding the nervous tissue, and extending into the fourth ventricle via the lateral aperture. No separate tumour masses were seen, in the cerebellum or elsewhere.

In the vertebral column there had been an extensive cervical laminectomy, and scar tissue was adherent to the dura mater. Vertebrae and intervertebral discs appeared normal, but there was a very prominent plexus of veins lying between the

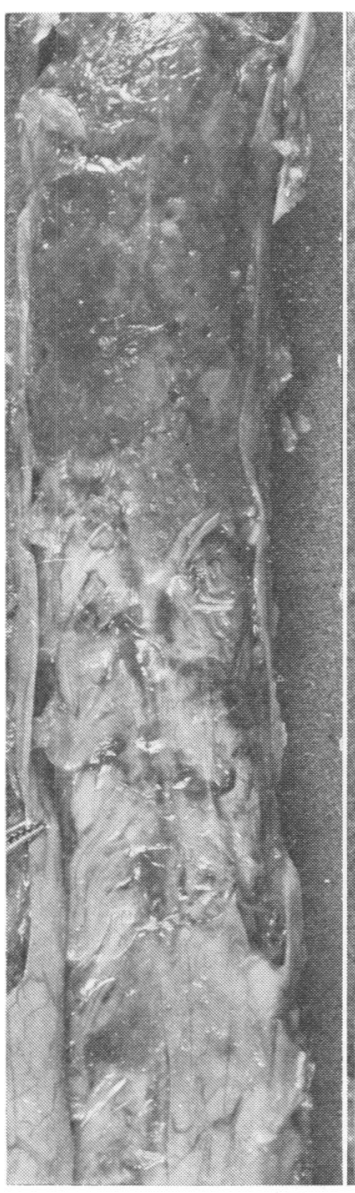

(a)

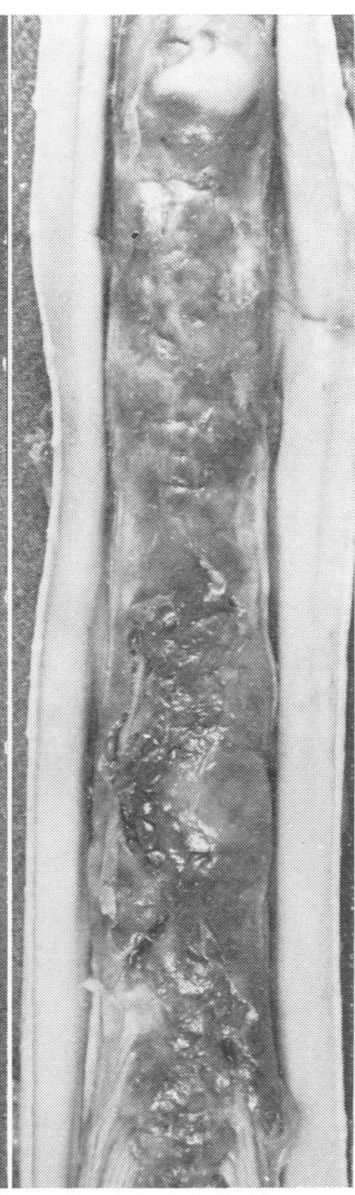

(b)
FIG. 3 Case 1. Spinal cord; posterior view of cervical (a) and lumbosacral (b) segments. lumbar vertebrae and the posterior longitudinal ligament.

The upper $5 \mathrm{~cm}$ of the spinal cord were invested in dark, firm tumour tissue, continuous with that surrounding the brainstem. The tumour lay mainly on the ventral surface of the cord, obliterating the subarachnoid space, and on section was seen to be eroding the cord; but there was no separate intramedullary tumour. The posterior columns of the entire cervical cord were shrunken and soft, and adherent to the dura mater in the area of

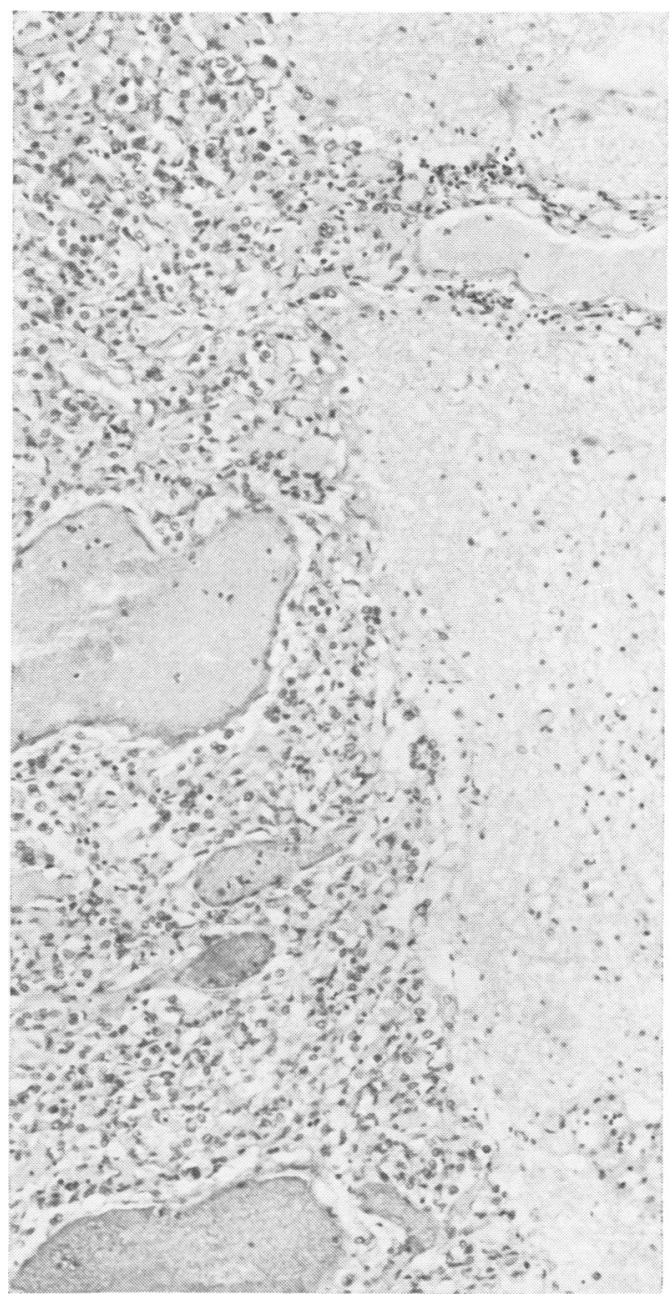

FIG. 4 Case 1. Surface of pons. Tumour (left) has obliterated the pia mater, and is eroding nervous tissue. A large vein is shared by the two tissues. $H$ and $E, \times 100$. 
laminectomy. The upper thoracic cord was swollen, and rather soft, and a 'plug' of necrotic cord tissue was present at the base of the posterior columns, extending down to the 4th thoracic segment. Below this, the cord appeared normal on section; but there was irregular thickening of the arachnoid mater, with nodules of tumour involving arachnoid and pia and nerve roots (Fig. 3). The roots of the cauda equina were matted together by dark fibrous tissue, with nodules on individual roots. At all levels, the spinal veins were tortuous and grossly dilated.

HISTOLOGY First biopsy (RI. S.H. 6728/64) The tumour was a typical capillary haemangioblastoma, with numerous small cystic spaces, and areas of xanthomatous change, attached to degenerate cerebellar cortex without intervening fibrous capsule. There were no mitotic figures or other signs of histological malignancy.

Second biopsy (R.I. S.H. 06391/73) This was very similar to the first biopsy specimen, but with more xanthomatous change. Some vessels in the tumour showed the type of endothelial hyperplasia commonly seen at the edges of malignant gliomas.

Necropsy (R.I. p.m. 1093/74) The tumour overlying the base of the brain and surrounding the spinal cord appeared identical with that seen in the first and second biopsy samples. No mitoses were seen, nor any signs of cellular anaplasia. The principal 'malignant' feature was that at many sites in the brainstem and cord the tumour had transgressed the pial surface, and was clearly invading nervous tissue (Fig. 4). The spread of tumour was far more extensive than appeared to the naked eye. In the right cerebellopontine angle, tumour was growing diffusely in the clefts between cerebellar folia, and in one region was replacing cerebellar cortex. Here, there was no way of deciding whether a primary tumour in the cortex had spread into the subarachnoid space, or whether the cortex had been invaded from outside. There was no recurrence at the site of the original operation. Diffuse subarachnoid spread was seen in the Sylvian fissures and chiasmatic cistern (Fig. 5), all around the brainstem, and at all seven levels of spinal cord which were examined. Sections of the scar from the cervical laminectomy showed tumour both inside and outside the spinal dura mater (Fig. 6). For the most part, the tumour masses which were visible to the naked eye could be regarded as local accentuations of \& diffuse spread. An exception to this was a smalf globular mass of tumour, attached by a shor $\mathbb{R}$ pedicle to the wall of the right lateral ventricle (Fig. 7).

A striking feature, especially in the sections of spinal cord, was the enormous dilatation of sur $\overrightarrow{0}$ face veins, even in places where no tumour tissuẹ के

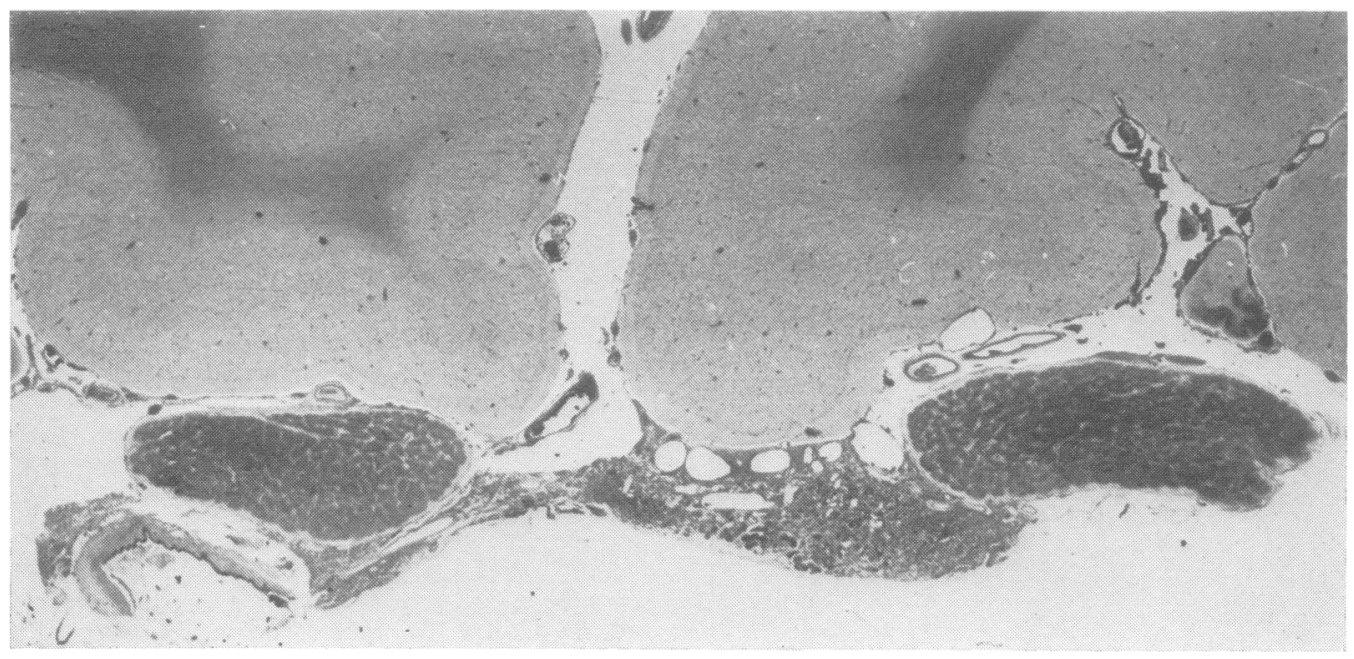

FIG. 5 Case 1. Gyri recti and optic nerves in coronal plane. Tumour tissue is adherent to cortex, optic nerves, and blood vessels. Myelin stain, $\times 5$. 


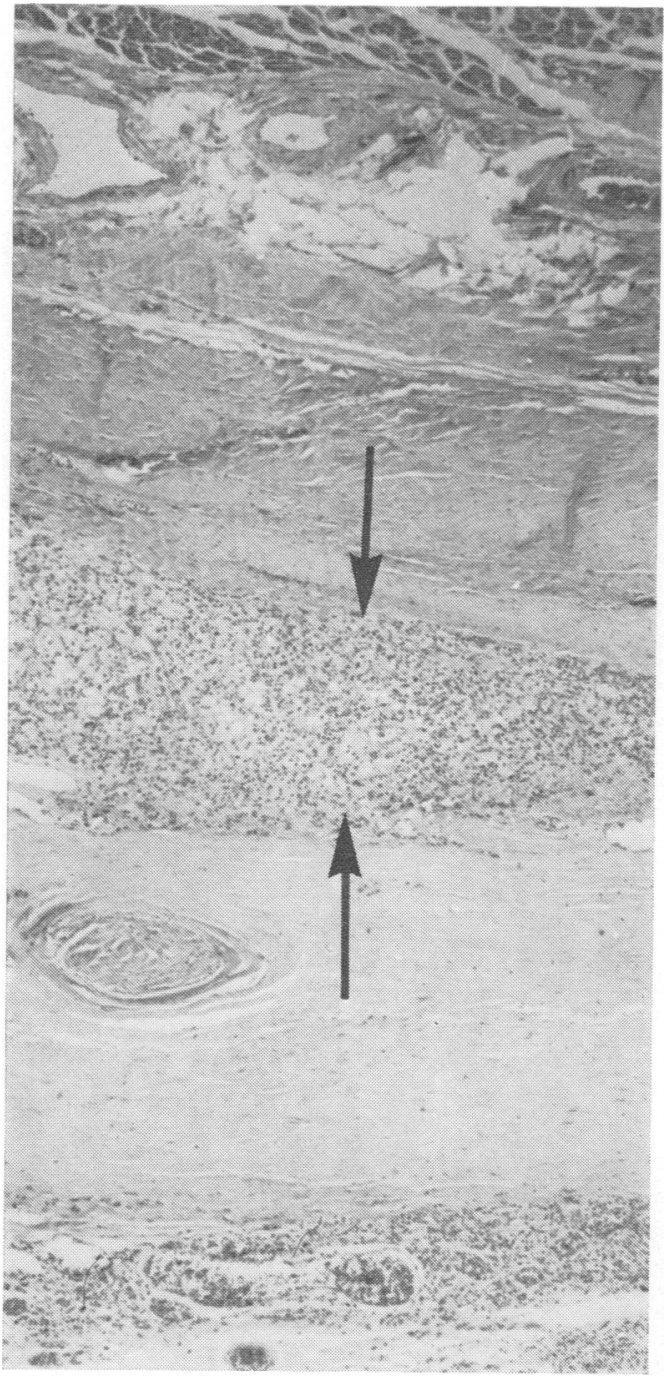

FIG. 6 Case 1. Section through laminectomy scar, showing (from above down): sacrospinalis muscle, scar tissue, tumour (arrows), dura mater, arachnoid mater with tumour. $H$ and $E, \times 42$.

was seen. Another was the frequent occurrence of dilated veins lying in tumour at one end and in nervous tissue at the other (Fig. 4).

There was necrosis, apparently due to ischaemia, of the posterior part of the lower cervical and upper thoracic cord segments. In the upper cervical region (Fig. 8) the whole cord showed ischaemic damage, with loss of nerve cells, but not total necrosis. Nerve roots, though heavily invested by tumour, were not invaded.

\section{CASE 2}

C. W. Male. Age at death, 53 years.

FIRST ADMISSION April 1960, to Bristol Neurosurgical Unit. He was admitted because of lassitude, headaches, vomiting, and difficulty in walking for 10 weeks. Examination showed drowsiness, disorientation, bilateral papilloedema, horizontal nystagmus, ataxia, and a tendency to fall to the right when standing or walking. A skull radiograph and a carotid angiogram were normal. Ventriculography revealed dilatation of the lateral and third ventricles, with the aqueduct pushed forward and the 4th ventricle pushed to the left. Posterior fossa craniectomy disclosed a cyst in the inferomedial part of the right cerebellar hemisphere, containing a plum-coloured nodule about $2 \frac{1}{2} \mathrm{~cm}$ diameter. This tumour was excised completely. Postoperative progress was smooth, but, because histology suggested that the tumour was malignant, radiotherapy was given to the posterior fossa. He was followed up as an outpatient and remained symptom-free for 14 years.

SECOND ADMISSION In July 1974 he was readmitted because of pain and stiffness in the neck, and weakness of the right arm and leg, for two months. Weakness was noted in all muscle groups in the right upper limb, and of the right leg. Tendon jerks were depressed on the left. There was no sensory abnormality.

Radiography and a myelogram showed marked cervical spondylosis with obstruction opposite C4-5 and C6-7 vertebrae, and areas of partial hold-up of contrast medium in the upper thoracic and lower lumbar regions. An EMI scan showed marked dilatation of the lateral ventricles, the third and fourth ventricles being normal. In July 1974, anterior cervical fusion of C4-5 and C6-7 vertebrae was performed. One month after operation he began to develop weakness and sensory impairment of the left upper and lower limbs, and progression of weakness of the right limbs. In September 1974, a cervical laminectomy from C2 to C6 was carried out, the dura mater was opened, the spinal cord was seen to be swollen, and at the level of the C5 vertebra there was an irregular, fleshy mass overlying the dorsal aspect of the cord, mainly on the right, and extending anteriorly. No attempt was made to remove the tumour, as there was no definable plane of cleavage. Over the subsequent four weeks the patient became tetraplegic, developed bronchopneumonia, and died. 
FIG. 7 Case 1.

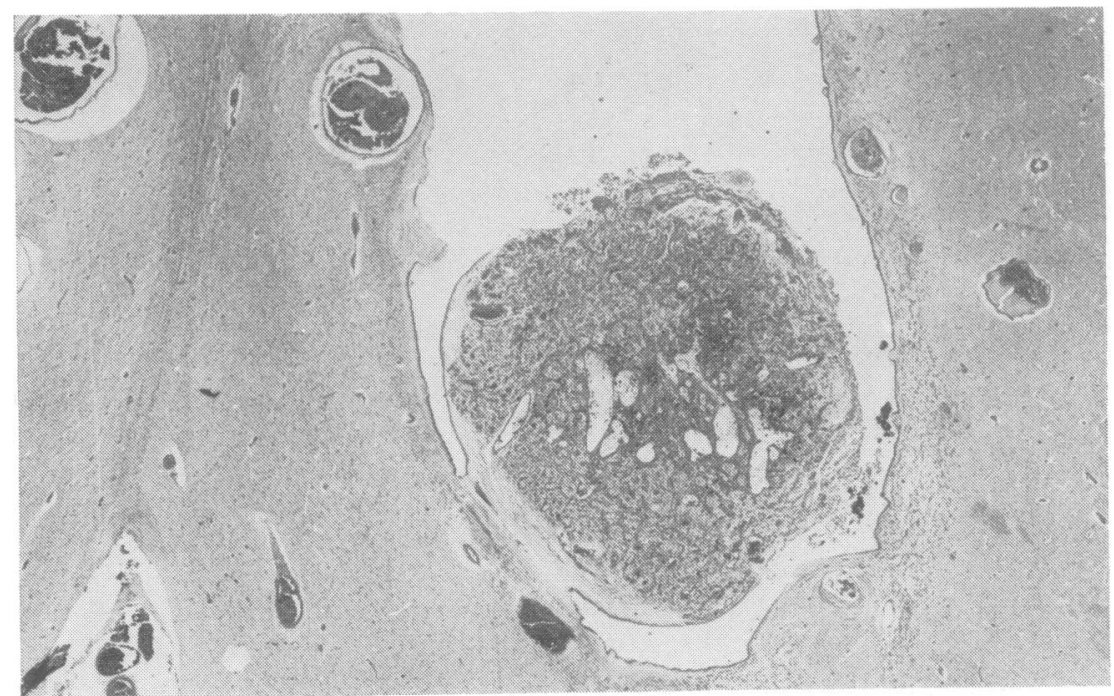

Anterior horn of right lateral ventricle. $A$ globular tumour mass is attached to the inferomedial surface of the ventricle by a short pedicle. $H$ and $E, \times 10$.

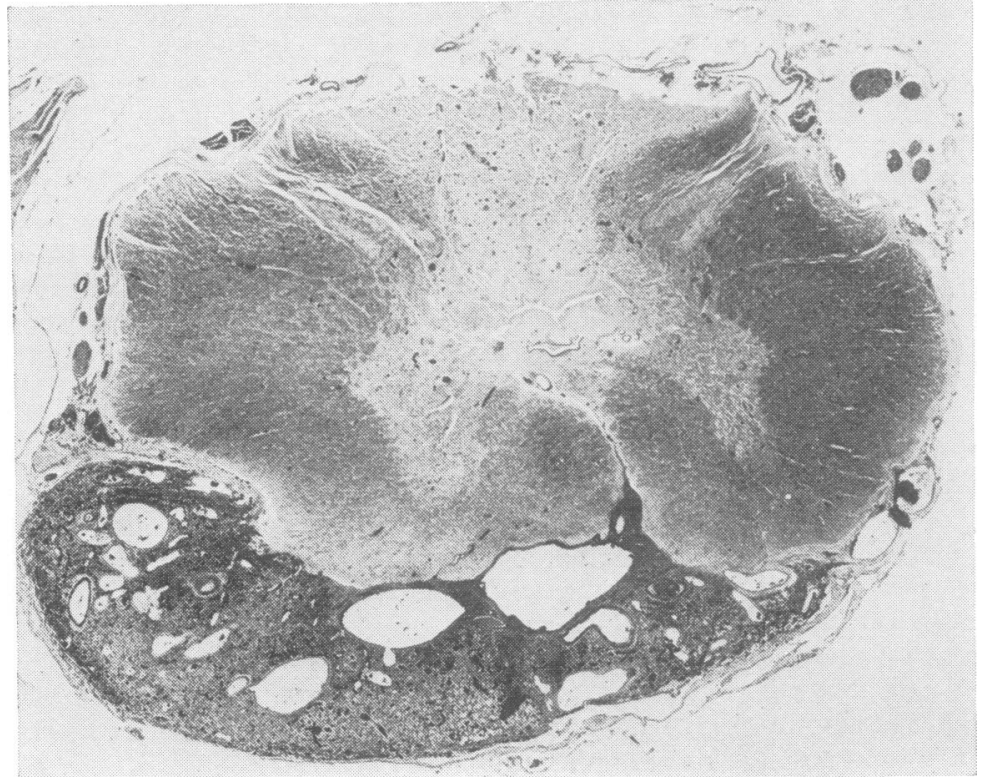

FIG. 8 Case 1. Section of cord at segmental level C3. In addition to the main tumour mass, there are tiny clusters of tumour cells attached to the pia mater at many points. The cord shows a mixture of ischaemic necrosis and Wallerian degeneration. Myelin stain, $\times 7$. 
NECROPSY FINDINGS There was extensive bilateral pneumonia. Otherwise, the only abnormality outside the nervous system was the presence of a few superficial yellowish nodules, less than $5 \mathrm{~mm}$ in diameter, in the cortex of both kidneys.

The brain $(1460 \mathrm{~g})$ appeared normal externally, except for some meningeal thickening at the base and old surgical damage to the right cerebellar hemisphere. Coronal slices of the cerebrum showed only slight dilatation of the ventricles. On slicing the cerebellum, two small, discrete tumours were seen, one in the superior vermis, the other in the cortex of the right lateral lobe. Each measured about $1 \mathrm{~cm}$ in diameter, was well circumscribed, and consisted of solid brownish tissue with large vascular spaces but no associated cyst (Fig. 9).

The spinal cord was swollen throughout the cervical and upper thoracic segments. From $\mathrm{C} 4$ to C6 there was a mass lying in front of and on the right side of the cord. Transverse sections showed dark red tumour, adherent to the cord, without a cleavage plane. At level C5 the tumour was $1 \mathrm{~cm}$ thick, and had compressed the cord into a crescent no more than $5 \mathrm{~mm}$ thick. Caudal to the tumour, the cord tissue was softened and contained a central syrinx. Below the upper thoracic region the cord looked normal, but many of the nerve roots, particularly in the lumbosacral region, were studded with small nodules, and the leptomeninges were diffusely thickened.
HISTOLOGY (1) Biopsy (F.B. 1046/60). The tumour was a capillary haemangioblastoma, containing small cysts and having a fine intercellular reticulin pattern, abutting on but not infiltrating cerebellar cortex. The presence of nuclear changes resembling mitotic figures, and of rather numerous giant cells with bizarre nuclei (Fig. 10), gave rise to the suspicion of malignancy, for which the patient received radiotherapy.

(2) Necropsy (N.P. 4399) The compressing tumour at segmental level C5 was a typical capillary haemangioblastoma, containing large and small cysts and some very dilated blood spaces (Fig. 11). It differed from the biopsy specimen in the absence of giant cells. No mitoses were seen. Nodules of similar tumour were adherent to pia or arachnoid mater, or wrapped around spinal nerve roots (Fig. 12); and microscopic collections of similar tumour cells were seen adhering to the meninges at all levels, from the chiasmatic cistern down to the cauda equina. The tumour at C5 segmental level had engulfed the pia mater of the cord, but had not invaded the nervous tissue, which merely showed distortion, local infarction, and oedema.

The lesions in the cerebellum had the appearance of primary tumours-that is, they were replacing cerebellar cortex, rather than invading it from the surface. Microscopically, they resembled

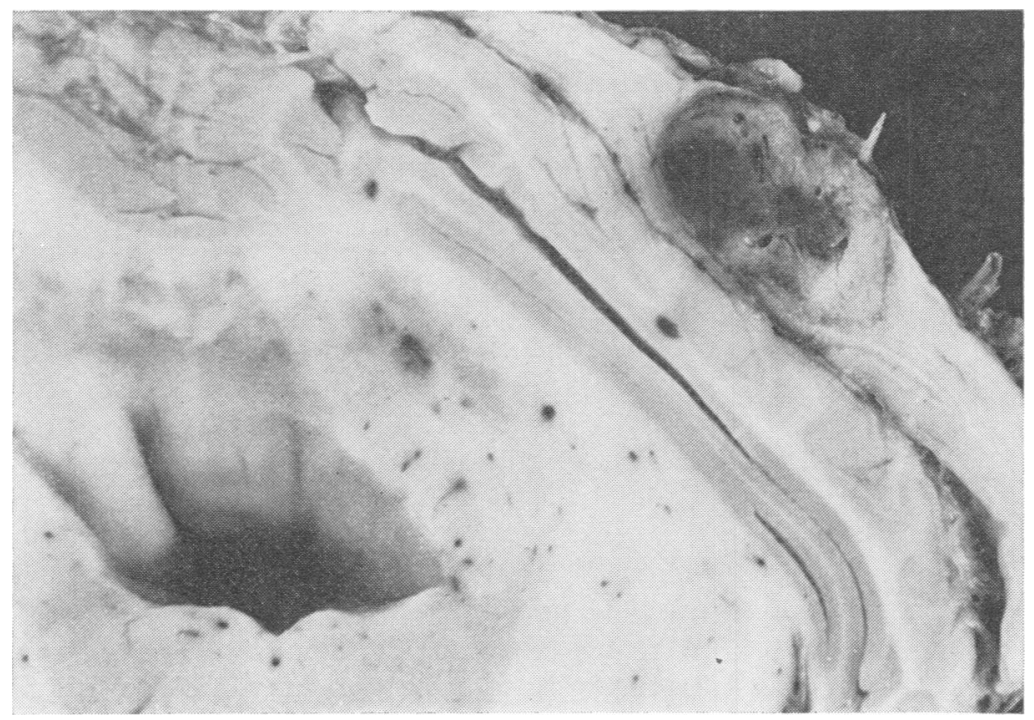

FIG. 9 Case 2. Small isolated haemangioblastoma in cerebellar cortex; probably a primary tumour. 


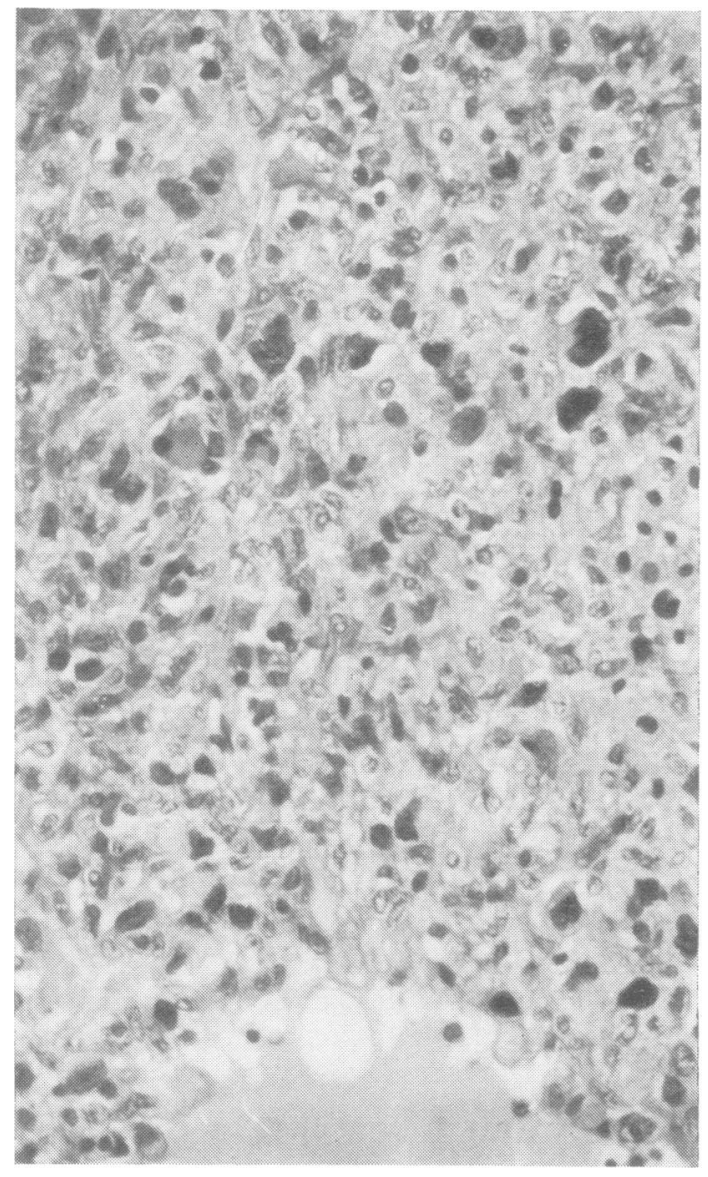

FIG. 10 Case 2. Tissue from original biopsy, showing nuclear irregularities, giant cells, and a small cyst (below). $H$. and $E, \times 260$.

the biopsy specimen rather than the spinal tumour, and contained giant cells, foamy cells, and traces of previous haemorrhages. No mitoses were seen.

At segmental levels $\mathrm{C} 4$ to $\mathrm{C} 6$, the cord was grossly compressed and distorted by tumour. Below this, as in case 1 , there was a partial ischaemic necrosis posteriorly and on the right. Lower segments showed Wallerian degeneration of the pyramidal tract on the right side.

The kidney lesions had the appearance of clearcelled adenomas (Fig. 13) without obvious malignant features. The possibility that they were metastatic deposits from the spinal tumour was consirlered, but dismissed.
DISCUSSION

We can find no parallel, in our experience or in the literature, for the malignant behaviour of the tumours in these two cases. There is no reasonable alternative, in either case, to the diagnosis of haemangioblastoma; the two types of tumour which have at times been confused with haemangioblastoma-renal carcinoma and angioblastic meningioma-can be firmly ruled out. To call them 'angiosarcomas' merely because of their malignant behaviour would solve no problems, as they do not differ microscopically from the general run of benign haemangioblastomas.

It is generally accepted that haemangioblastomas are innocent tumours composed of capillary and other blood vessels, mixed with interstitial cells, the origin of which is still debated (Spence and Rubinstein, 1975); that they occur most commonly in the cerebellum, some $\frac{0}{\mathbb{Q}} \frac{\mathrm{or}}{\mathrm{v}}$ times in the spinal cord or spinal meninges, and very rarely above the tentorium; that they may be multiple, especially in cases with ar family history of the von Hippel-Lindau syn- -0 drome (Olivecrona, 1952); and that they are sometimes associated with cysts or tumours in unrelated viscera, with angiomatosis (or haemangioblastoma) of the retina, and with polycythaemia.

Malignant spread, distant metastasis, and subarachnoid 'seeding' are said not to occur (Russell and Rubinstein, 1971; Rubinstein, 1972). Recurrences after apparently complete removal have been reported by Pennybacker (1954), Palmer (1972), and others. In Pennybacker's first case, a recurrent tumour had grown to occupy most of the posterior fossa, become attached to the tentorium, and spread through the craniectomy scar into the neck muscles and skin, but diffuse spread and disseminated seeding were not observed. In many cases, it is hard to decide whether or not the 'recurrent' tumour is an independent growth. If the 'recurrence' is at a distant site, it is generally assumed to be an independent tumour; if it is at the original operation site, it is assumed that the removal must have been incomplete. This was thought to have occurred, for instance, in the case reported by Vinas and 


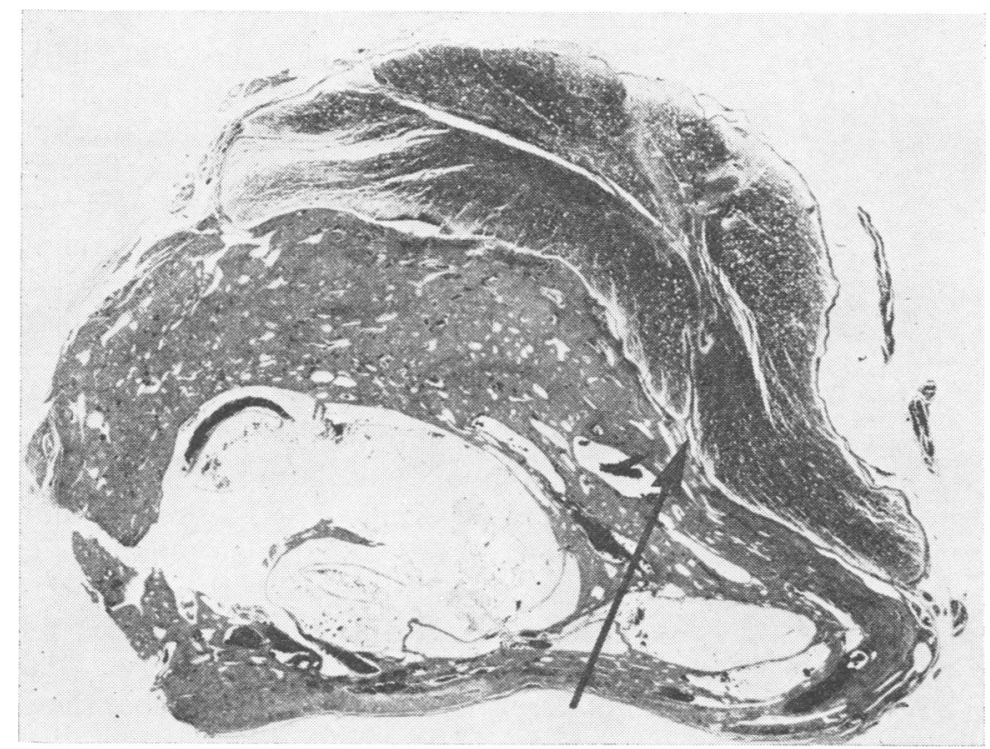

FIG. 11 Case 2. Section of fifth cervical cord segment, distorted by tumour. Arrow indicates anterior median sulcus of cord. Myelin stain, $\times 5$.

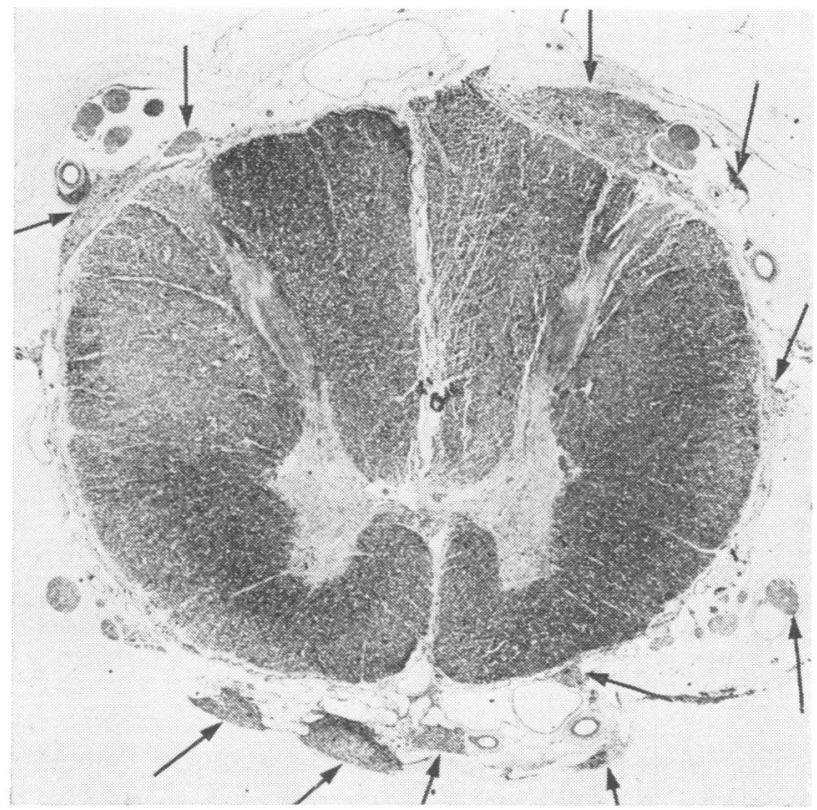

FIG. 12 Case 2. Upper thoracic cord segment. There is degeneration of descending fibres on the right (left side of picture). Arrows indicate tumour tissue attached to nerve roots and leptomeninges. Myelin stain, $\times 8$. 


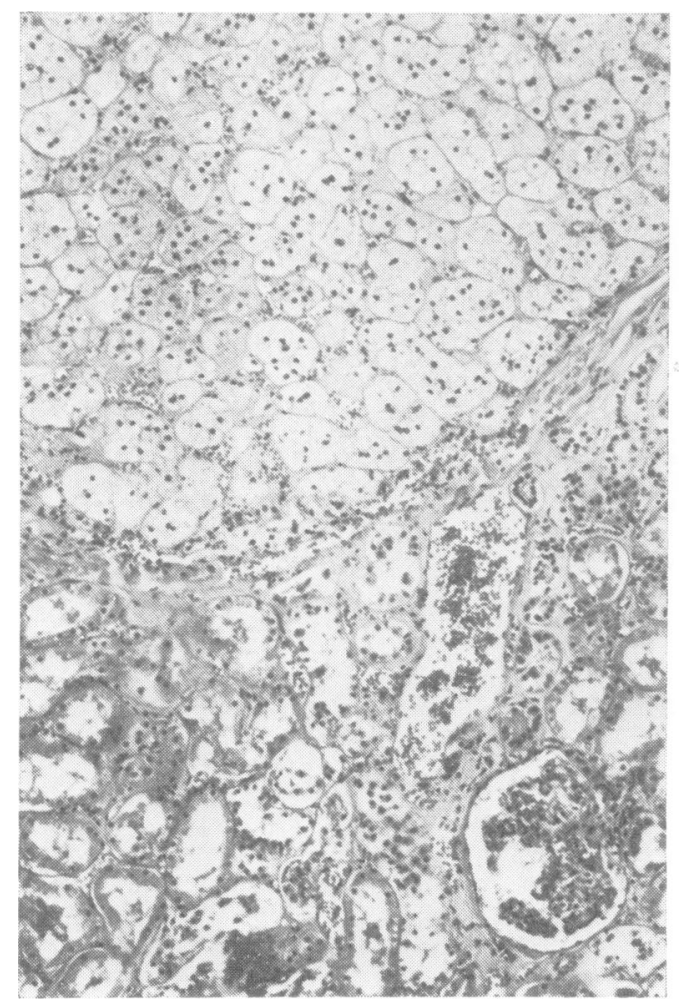

FIG. 13 Case 2. Clear-celled adenoma in renal cortex. $H$ and $E, \times 100$.

Horrax (1956) of recurrence in situ 22 years after the first operation.

In our two cases, there was no local recurrence of the original tumour. In case 2 , there were two additional tumours, apparently primary, in the cerebellar cortex. We found it impossible to decide whether the tumour at segmental level $\mathrm{C5}$ in case 2, and the large spinal and brainstem tumours in case 1 , were independent growths or the result of malignant spread from a single source; but examination of the smaller meningeal collections of tumour tissue, with a distribution closely resembling that seen in meningeal carcinomatosis, left no doubt that malignant spread had occurred, and that we were not looking at innumerable primary tumours. In neither case was it possible to decide how much of the superficial tumour had arisen by continuous spread, and how much by 'seeding' in the subarachnoid space; but there can be little doubt that in case 1 the isolated pedunculated tumour in the lateral ventricle (Fig. 8) is a seedling, presumably carried upwards in a retrograde flow of CSF caused by the presence of a ventriculoatrial shunt. The finding of extradural tumour in the laminectomy scar in case 1 (Fig. 7) is sufficient evidence that detached tumour fragments were capable of implantation. If this is so, one is bound to wonder whether some of the 'multiple' tumours described in the literature may not in fact have been seedlings.

Both cases-especially case 1 - showed enormous expansion of superficial small veins draining either central nervous tissue or nerve roots. How this change comes about is not clear; but it is not peculiar to these cases. Dilatation of cerebellar veins in the neighbourhood of a haemangioblastoma is commonly observed at operation, and demonstrated angiographically (Olivecrona, 1952).

Regarding the management of such cases as these, there is little to be said. Both, in the later stages, were well beyond the scope of surgical treatment. In case 1 there was no evidence, either clinical or histological, that radiotherapy had exerted any effect on the tumour tissue.

In reviewing the slides of the original biopsies, we have not observed any histological feature which could be used to predict similar malignant spread in other cases of haemangioblastoma.

We wish to thank Dr D. L. Stevens, Mr D. G. Phillips and Mr C. B. T. Adams for permission to use their case records.

\section{REFERENCES}

Olivecrona, H. (1952). The cerebellar angioreticulomas. Journal of Neurosurgery, 9, 317-330.

Palmer, J. J. (1972). Haemangioblastomas: a review of 81 cases. Acta Neurochirurgica, 27, 125-148.

Pennybacker, J. (1954). Recurrence in cerebellar haemangiomas. Zentrablatt für Neurochirurgie, 12, 63-73.

Rubinstein, L. J. (1972). Tumours of the Central Nervous System. Armed Forces Institute of Pathology; Washington, D.C. 
Russell, D. S., and Rubinstein, L. J. (1971). Pathology of Tumours of the Nervous System, 3rd edn. Arnold, London.

Spence, A. M., and Rubinstein, L. J. (1975). Cerebellar capillary hemangioblastoma: its histogenesis studied by organ culture and electron microscopy. Cancer, 35, 326-341.

Vinas, F. J., and Horrax, G. (1956). Recurrence of cerebellar hemangioblastoma after 22 years-operation and recovery. Journal of Neurosurgery, 13, 641-646. 